\title{
Polynomial Asymptotic Estimates of Gegenbauer, Laguerre, and Jacobi Polynomials
}

Nico M. Temme Professor, Center for Mathematics and Computer Science, Amsterdam, The Netherlands

\section{INTRODUCTION}

We discuss asymptotic forms of $C_{n}^{\gamma}(x), L_{n}^{\alpha}(x), P_{n}^{(\alpha, \beta)}(x)$, the Gegenbauer, Laguerre and Jacobi polynomials. The asymptotic behavior of these classical orthogonal polynomials has been the subject of several investigations. The research usually concentrates on the case that the degree $n$ of the polynomial is the large parameter, and for all classical orthogonal polynomials the asymptotic behavior is well established now. Inside the domain of the zeros of the polynomial the behavior can be described in terms of elementary functions, such as trigonometric functions. In the domain where the transition from oscillatory to monotonic behavior occurs, familiar higher transcendental functions can be used as estimates. For example, the "first" zeros of the Jacobi polynomial can be approximated in terms of the zeros of the $J$-Bessel function. In Szegö (1958) several classical results can be found. For Jacobi polynomials he gives an estimate of 
"Hilb's type", which is an approximation in terms of the $J$-Bessel function. In earlier papers, however, Szegö already gave more extensive expansions for Legendre and Jacobi polynomials, again in terms of the $J$-Bessel function. See ASKEY (1982).

More recent publications give uniform asymptotic expansions in larger domains for the argument, and also more terms in the expansion. ERDÉLYI (1960) derived leading terms of the expansion for Laguerre polynomials in terms of Bessel and Airy functions, and quite recently FRENZEN \& Wong (1988) constructed complete expansions for this class of polynomials. ELLrotT (1971) treated the Jacobi polynomials essentially outside the domain of orthogonallity, giving a full expansion in terms of modified Bessel functions; in FrENZEN \& WONG (1985) a uniform expansion for the Jacobi polynomials is given in terms of $J$-Bessel functions, complete with error bounds for the remainder.

As mentioned earlier, the degree $n$ of the polynomials is usually considered as the large parameter in the asymptotic expansions. In many existing cases, however, the asymptotic variable includes additional parameters as well. For instance, in ERDÉLYI (1960) and FRENZEN \& WONG (1988) a natural choice for the large parameter of the Laguerre polynomial $L_{n}^{\alpha}(x)$ is $\kappa=n+(\alpha+1) / 2$, although the parameter $\alpha$ (and hence the order of the comparison function $\left.J_{\alpha}(z)\right)$ is kept fixed in their analysis. In Temme (1986) three different cases are considered for large values of $\kappa$. Depending on the value of $x$ and the size of $\alpha / n$, a Bessel function, a Hermite polynomial, or an Airy function is used for the Laguerre polynomials.

In the case of Jacobi polynomials $P_{n}^{(\alpha, \beta)}(x)$, a convenient choice is $\kappa=n+(\alpha+\beta+$ 1)/2, see Frenzen \& Wong (1985). See also Elliott (1971) and Nestor (1984). In this paper we consider the same asymptotic variable $\kappa$, now with the possibility that $\alpha$ and/or $\beta$ dominate $n$, and with $x$ inside the domain of orthogonallity. We use the Gegenbauer polynomial and the Laguerre polynomial as new estimates for the Jacobi polynomial.

Some of the results on orthogonal polynomials in this paper follow from earlier, sometimes more general, results in the literature. The Laguerre polynomial is in fact a special case of the Whittaker, or confluent hypergeometric function. For this class of functions many recent results are available, for instance, OLVEr(1980), and very recently DUNSTER(1989). On the other hand, the Jacobi polynomial and the Gegenbauer polynomial are special cases of the Gauss hypergeometric function, for which class rather few interesting uniform expansions have been investigated.

Recently, other investigations on orthogonal polynomials with several large parameters have been published, but the approach is quite different from ours. LI-CHEN 
Chen \& IsmaIL (1989) investigated the asymptotics of $P_{n}^{(\alpha+a n, \beta+b n)}(x)$ as $n \rightarrow \infty$, and $a, b, \alpha, \beta, x$ remain fixed. The approximants are in terms of elementary functions, and the approach is based on Darboux's method applied on a generating function. They also considered the Laguerre polynomial $L_{n}^{\alpha+a n}(b+x n)$ for large values of $n$. Asymptotics of Jacobi and Laguerre polynomials with similar arrangement of parameters occur in work on incomplete polynomials of SAFF \& VARga (1979), MOAK, SAFF \& VARGA (1979), and MHASKar \& SAFF (1984). In the second paper the distribution of zeros of that type of Jacobi polynomial is considered.

In the present approach, the estimates of the orthogonal polynomials are obtained by using differential equations, and Liouville Green transformations thereof. This technique is extensively developed by Frank Olver. Some of the results follow from his earlier work on, for instance, Whittaker functions. The proofs of the new estimates of Jacobi polynomials will be given elsewhere. Another interesting problem is how to obtain similar estimates by using integral representations of the polynomials. We intend to consider also this point in future publications.

\subsection{The Liouville-Green transformation}

The techniques used in this paper are based on the Liouville-Green (LG) transformation. Starting point is a differential equation of the form

$$
W^{\prime \prime}=\left[\kappa^{2} p(x)+q(x)\right] W,
$$

defining the orthogonal polynomial, where $\kappa$ is a large parameter, a simple function of the degree and other parameters, but independent of $x$. Formally we proceed as follows. A transformation of both the dependent and independent variable is introduced by writing

$$
W(x)=\sqrt{\dot{x}} w(\xi),
$$

where $\xi=\xi(x)$, and the dot indicates differentiation with respect to $\xi$. Then (1.1) becomes

$$
\ddot{w}=\left[\kappa^{2} \dot{x}^{2} p(x)+\psi(\xi)\right] w,
$$

where

$$
\psi(\xi)=\dot{x}^{2} q(x)+\sqrt{\dot{x}} \frac{d^{2}}{d \xi^{2}} \frac{1}{\sqrt{\dot{x}}} .
$$

Depending on the nature of the function $p$ and on the asymptotic phenomena to be investigated, the function $\xi(x)$ is defined by replacing $\dot{x}^{2} p(x)$ by a simple rational function $r(\xi)$. That is, we write

$$
\dot{x}^{2} p(x)=r(\xi)
$$


and the new differential equation becomes

$$
\ddot{w}=\left[\kappa^{2} r(\xi)+\psi(\xi)\right] w .
$$

The function $r(\xi)$ is chosen such that it mimics features of $p(x)$ that are essential for the asymptotic behavior of $W(x)$. As a rule, when we take $\psi=0,(1.6)$ reduces to the differential equation of a known special function that plays the part of a basic approximant in the asymptotic estimate.

For example, for the orthogonal polynomials on the interval $[-1,1], p$ has two simple zeros $x_{1}, x_{2}$ in this interval, and $p$ is negative in $\left(x_{1}, x_{2}\right)$. Then the zeros of the orthogonal polynomial occur in the same interval. Under the influence of some parameters we may have the situation that $x_{1} \rightarrow x_{2}$. When no other zeros or poles of $p$ are nearby the interval $\left(x_{1}, x_{2}\right)$, one may consider for that particular case the transformation (1.5) in the form

$$
\dot{x}^{2} p(x)=\xi^{2}-\rho^{2}
$$

where $\rho$ depends on $x_{1}, x_{2}$. When $r(\xi)=\xi^{2}-\rho^{2}$ and $\psi=0$, equation (1.6) reduces to the differential equation for the parabolic cylinder function. Under further special circumstances, this function reduces to a Hermite polynomial.

In this paper we transform the differential equations defining the classical orthogonal polynomials $P_{n}^{(\alpha, \beta)}(x), L_{n}^{\alpha}(x), C_{n}^{\gamma}(x)$, the Jacobi, Laguerre and Gegenbauer polynomials, to a form in which another orthogonal polynomial acts as a basic approximant, for instance a Hermite polynomial. In these cases, the $x$-domain is the complete domain of orthogonallity. We also mention estimates in which other comparison functions occur, and which describe the transition of the oscillatory to the monotonic regions. In all cases we concentrate on real values of the parameters.

\section{Gegenbauer polynomials}

The Gegenbauer polynomials, or ultraspherical polynomials, can be defined by the generating function

$$
\left(1-2 x z+z^{2}\right)^{-\gamma}=\sum_{n=0}^{\infty} C_{n}^{\gamma}(x) z^{n}, \quad-1<x<1, \quad|z|<1 .
$$

An explicit representation is

$$
C_{n}^{\gamma}(x)=\frac{1}{\Gamma(\gamma)} \sum_{k=0}^{\lfloor n / 2\rfloor} \frac{(-1)^{k} \Gamma(\gamma+n-k)}{k !(n-2 k) !}(2 x)^{n-2 k},
$$


where $\lfloor m\rfloor$ is the integer number satisfying $\lfloor m\rfloor \leq m<\lfloor m\rfloor+1$, with $m \in \mathbb{R}$. These polynomials satisfy the differential equation

$$
\left(1-x^{2}\right) y^{\prime \prime}-(2 \gamma+1) x y^{\prime}+n(n+2 \gamma) y=0
$$

A simple transformation

$$
W(x)=\left(1-x^{2}\right)^{(2 \gamma+1) / 4} C_{n}^{\gamma}(x)
$$

gives for $W$ the equation

$$
\left(1-x^{2}\right) W^{\prime \prime}+\left[(n+\gamma)^{2}+\frac{2+4 \gamma-4 \gamma^{2}+x^{2}}{4\left(1-x^{2}\right)}\right] W=0 .
$$

This can be written in the form

$$
W^{\prime \prime}=\kappa^{2} \frac{x^{2}-x_{0}^{2}}{\left(1-x^{2}\right)^{2}} W-\frac{x^{2}+3}{4\left(1-x^{2}\right)^{2}} W
$$

with

$$
\kappa=n+\gamma, \quad x_{0}^{2}=\frac{\kappa^{2}-\left(\gamma-\frac{1}{2}\right)^{2}}{\kappa^{2}} .
$$

We assume that $\gamma>1 / 2$, and $n=0,1,2, \ldots ; x_{0}$ is the positive number defined by the above equation. We have $x_{0} \in[0,1]$. For the asymptotic problem we assume that $\kappa$ is large.

Especially interesting is the behavior of the polynomials when $x$ crosses the values $\pm x_{0}$ (turning points of (2.5)) and \pm 1 (singular points). When $\gamma>>n$ the turning points (and all zeros of the Gegenbauer polynomial) tend to zero and coalesce. When $n>>\gamma$ the turning points coalesce with the singularities at \pm 1 . In both cases, the asymptotic behavior of the polynomials cannot be described in terms of elementary functions.

The Gegenbauer polynomial is a function of three variables. In the asymptotic problem we try to find approximating functions of at most two variables. This gives a proper reduction of variables, as pointed out by OLvER (1975-B). It is not possible to construct one approximation of two variables with which we can describe the behavior of the polynomials as $\kappa \rightarrow \infty$, uniformly with respect to $x$ on the entire real axis. For the transition near $x= \pm 1$ we need a $J$-Bessel function as approximant. 


\subsection{Estimation in terms of a Hermite polynomial}

Consider as a simpler form of (2.5), especially if $\kappa$ is large and $x$ is bounded away from \pm 1 , the equation

$$
u^{\prime \prime}=\kappa^{2}\left(x^{2}-x_{0}^{2}\right) u
$$

with solution

$$
u(x)=D_{\nu}(x \sqrt{2 \kappa}), \quad 2 \nu+1=\kappa x_{0}^{2}
$$

where $D_{\nu}(x)$ is one of the Weber parabolic cylinder functions. This function is related to the Hermite polynomial. We have for integer values of $n$

$$
H_{n}(z)=2^{n / 2} e^{z^{2} / 2} D_{n}(z \sqrt{2})
$$

where the Hermite polynomial is given by

$$
H_{n}(x)=n ! \sum_{k=0}^{\lfloor n / 2\rfloor} \frac{(-1)^{k}}{k !(n-2 k) !}(2 x)^{n-2 k} .
$$

Observe that when $n<<\gamma$ the quantity $\nu$ behaves like $\nu=n+\mathcal{O}\left(\gamma^{-1}\right)$. So it appears that the "first approximation" (2.7) does not exactly yield a Hermite polynomial.

In this section we construct a first approximation to (2.5) based on a LG transformation, which exactly reduces to the equation for the Hermite polynomial. Then, by using the theorems of OLVER (1975-A), the asymptotic estimation of the Gegenbauer polynomials can be described in more detail. This gives an asymptotic interpretation of the well-known limit relation

$$
\lim _{\gamma \rightarrow \infty} \gamma^{-n / 2} C_{n}^{\gamma}(x / \sqrt{\gamma})=\frac{1}{n !} H_{n}(x),
$$

which can be verified by considering (2.2) and (2.9), and the limit of each coefficient of the Gegenbauer polynomial.

The points $\pm x_{0}$ are two turning points of (2.5). By hypothesis $x_{0}$ is bounded away from the singularities \pm 1 . In this subsection the conditions on $x, x_{0}$ read

$$
x \in(-1,1), \quad x_{0} \in[0, b], \quad \text { with } b \text { fixed in }(0,1)
$$

The condition $x_{0} \leq b$ implies

$$
\gamma \geq \frac{\frac{1}{2}+n \sqrt{1-b^{2}}}{1-\sqrt{1-b^{2}}}
$$

Usually, $n$ is considered as the large parameter; from the above condition and $\kappa \rightarrow \infty$ it follows that $\gamma \rightarrow \infty$, and that $n$ may be large, but not larger than $\mathcal{O}(\gamma)$. 
We now transform (2.5) into an equation with as dominant part an equation of the form (2.7). Considering the basic steps outlined in (1.1) - (1.6), we perceive that the appropriate LG transformation reads

$$
\left(\eta^{2}-\rho^{2}\right)\left(\frac{d \eta}{d x}\right)^{2}=\frac{x^{2}-x_{0}^{2}}{\left(1-x^{2}\right)^{2}}, \quad W(x)=\sqrt{\dot{x}} w(\eta),
$$

where the dot indicates differentiation with respect to $\eta$, and the non-negative number $\rho$ is defined by

$$
\int_{-\rho}^{\rho} \sqrt{\rho^{2}-\eta^{2}} d \eta=\int_{-x_{0}}^{x_{0}} \frac{\sqrt{x_{0}^{2}-x^{2}}}{1-x^{2}} d x .
$$

Evaluation of both integrals yields

$$
1 / 2 \pi \rho^{2}=\pi\left(1-\sqrt{1-x_{0}^{2}}\right)
$$

It is convenient to introduce parameters $\nu, \mu$ by writing

$$
x_{0}=\sin \mu, \quad \nu=\cos \mu, \quad \mu \in[0,1 / 2 \pi]
$$

Then we have, using (2.6),

$$
\rho=\sqrt{\frac{2 n+1}{\kappa}}=2 \sin (1 / 2 \mu) .
$$

The relation between $\eta$ and $x$ is one-to-one, with the points $-x_{0}$ and $x_{0}$ corresponding respectively to $-\rho$ and $\rho$. On taking the positive square root in the first of (2.11) and integrating, we obtain

$$
\begin{array}{cc}
\int_{-\infty}^{\eta} \sqrt{\bar{\eta}^{2}-\rho^{2}} d \bar{\eta}=\int_{-1}^{x} \frac{\sqrt{\bar{x}^{2}-x_{0}^{2}}}{1-\bar{x}^{2}} d \bar{x}, & -1<x \leq-x_{0} \\
\int_{-\rho}^{\eta} \sqrt{\rho^{2}-\bar{\eta}^{2}} d \bar{\eta}=\int_{-x_{0}}^{x} \frac{\sqrt{x_{0}^{2}-\bar{x}^{2}}}{1-\bar{x}^{2}} d \bar{x}, & -x_{0} \leq x \leq x_{0} \\
\int_{\rho}^{\eta} \sqrt{\bar{\eta}^{2}-\rho^{2}} d \bar{\eta}=\int_{x_{0}}^{x} \frac{\sqrt{\bar{x}^{2}-x_{0}^{2}}}{1-\bar{x}^{2}} d \bar{x}, & x_{0}<x<1 .
\end{array}
$$

From these relations it follows that $\eta$ is an increasing function of $x$ with $\eta=-\infty$ and $+\infty$ corresponding to $x=-1$ and +1 , respectively.

If $x_{0} \neq 0$, the relation between $x$ en $\eta$, defined in (2.11) is given by (i) $-\rho \leq \eta \leq \rho, \quad-x_{0} \leq x \leq x_{0}$ :

$$
1 / 2\left[\eta \sqrt{\rho^{2}-\eta^{2}}+\rho^{2} \arcsin \frac{\eta}{\rho}\right]=\arcsin \frac{x}{x_{0}}-\nu \arctan \frac{\nu x}{\sqrt{x_{0}^{2}-x^{2}}}
$$


(ii) $\eta \geq \rho, \quad x_{0} \leq x<1$ :

$$
1 / 2\left[\eta \sqrt{\eta^{2}-\rho^{2}}-\rho^{2} \operatorname{arccosh} \frac{\eta}{\rho}\right]=-\operatorname{arccosh} \frac{x}{x_{0}}+\nu \operatorname{arctanh} \frac{\sqrt{x^{2}-x_{0}^{2}}}{\nu x}
$$

(iii) $\eta \leq-\rho, \quad-1<x \leq-x_{0}$ :

$$
-1 / 2\left[\eta \sqrt{\eta^{2}-\rho^{2}}+\rho^{2} \operatorname{arccosh} \frac{-\eta}{\rho}\right]=-\operatorname{arccosh} \frac{-x}{x_{0}}-\nu \operatorname{arctanh} \frac{\sqrt{x^{2}-x_{0}^{2}}}{\nu x} .
$$

Alternatively, when $x_{0}=0$, so that $\rho=0$, we find

$$
1 / 2 \eta^{2}=-1 / 2 \ln \left(1-x^{2}\right), \quad \text { or } \quad \eta=\sqrt{-\ln \left(1-x^{2}\right)},
$$

with $\operatorname{sign}(\eta)=\operatorname{sign}(x)$. Whether or not $x_{0}=0$, the differential equation (2.5) transforms into the form (1.6):

$$
\ddot{w}=\kappa^{2}\left(\eta^{2}-\rho^{2}\right) w+\psi(\eta) w
$$

where

$$
\psi(\eta)=-\dot{x}^{2} \frac{x^{2}+3}{4\left(1-x^{2}\right)^{2}}+\sqrt{\dot{x}} \frac{d^{2}}{d \eta^{2}} \frac{1}{\sqrt{\dot{x}}} .
$$

By carrying out the differentiations by means of the first of (2.11), we arrive at the formula

$$
\psi(\eta)=\frac{3 \eta^{2}+2 \rho^{2}}{4\left(\eta^{2}-\rho^{2}\right)^{2}}+\frac{\left(\eta^{2}-\rho^{2}\right)\left(1-x^{2}\right)}{4\left(x^{2}-x_{0}^{2}\right)^{3}}\left(4 x^{2} x_{0}^{2}-3 x^{2}-2 x_{0}^{2}+x_{0}^{4}\right) .
$$

The right-hand side is to be replaced by its limiting form when $\eta= \pm \rho$. It follows from Olver's theory that $\psi$ is a continuous function of $\eta \in \mathbb{R}$, but for this special case we can prove that $\eta$ is an analytic function of $x \in(-1,1)$ and $x_{0} \in[0,1)$, and that $\psi$ is an analytic function of $\eta$. For instance, at $\eta=0$ and as $x_{0} \rightarrow 0$, we have $\psi(0)=-1 / 32 x_{0}^{2}+\mathcal{O}\left(x_{0}^{4}\right)$.

We need to know the asymptotic behavior of $x(\eta)$ as $\eta \rightarrow \pm \infty$. From (2.15) we derive that

$$
\begin{aligned}
& 1 / 2 \eta^{2}-1 / 4 \rho^{2}-1 / 2 \rho^{2} \ln \left(\frac{2 \eta}{\rho}\right)+\mathcal{O}\left(\eta^{-2}\right)=1 / 2 \nu \ln \left(2 \cot ^{2} \mu\right) \\
& -\operatorname{arccosh}\left(\frac{1}{x_{0}}\right)-1 / 2 \nu \ln (1-x)+\frac{1+x_{0}^{2}}{4 \nu}(1-x)+\mathcal{O}\left[(1-x)^{2}\right]
\end{aligned}
$$

as $\eta \rightarrow+\infty$, with a similar relation with $\eta \rightarrow-\infty$. It follows that

$$
1-|x|=c\left(x_{0}\right)|\eta|^{\rho^{2} / \nu} e^{-\eta^{2} / \nu}[1+o(1)], \quad \text { as } \quad \eta \rightarrow \pm \infty,
$$


where $c\left(x_{0}\right)$ does not depend on $\eta$, and is given by

$$
c\left(x_{0}\right)=\exp \frac{\rho^{2} / 2+\nu \ln \left(2 \cot ^{2} \mu\right)-2 \operatorname{arccosh}\left(1 / x_{0}\right)+\rho^{2} \ln (2 / \rho)}{\nu} .
$$

Further,

$$
\psi(\eta)=\mathcal{O}\left(\frac{1}{1+\eta^{2}}\right), \quad \eta \in \mathbb{R}
$$

uniformly with respect to $x_{0} \in[0, b]$.

\subsection{Identification of solutions}

As remarked above, the solutions of

$$
\ddot{w}_{0}=\kappa^{2}\left(\eta^{2}-\rho^{2}\right) w_{0}
$$

are parabolic cylinder functions. By considering the behavior of the Gegenbauer polynomial and that of the two solutions of the differential equation for parabolic cylinder functions, it follows that only the function $D_{\nu}(z)$ should be used as approximant. As in (2.7) we have, using (2.13)

$$
w_{0}(\eta)=D_{\nu}(\eta \sqrt{2 \kappa}), \quad 2 \nu+1=\kappa \rho^{2}=2 n+1
$$

It follows that the parabolic cylinder function reduces to a Hermite polynomial. Using $(2.8)$, we obtain as a solution of $(2.20)$

$$
w_{0}(\eta)=2^{-n / 2} e^{-\kappa \eta^{2} / 2} H_{n}(\eta \sqrt{\kappa}) .
$$

We obtain for the complete equation (2.17)

$$
w(\eta)=2^{-n / 2} e^{-\kappa \eta^{2} / 2} H_{n}(\eta \sqrt{\kappa})+\varepsilon(\eta) .
$$

Since our function $\psi$ of (2.17) satisfies the conditions of Theorem I of OLVER (1975-A, $\S 6$ ), we can infer that $|\varepsilon(\eta)|$ is small compared with $\left|w_{0}(\eta)\right|$, except in the neighborhoods of the zeros of this function, where $|\varepsilon(\eta)|$ is small compared with the envelope of $\left|w_{0}(\eta)\right|$. This conclusion holds uniformly with respect to $\eta \in \mathbb{R}, x_{0} \in[0, b]$. It is also possible to construct bounds for $|\varepsilon(\eta)|$. In terms of the Gegenbauer polynomial we obtain

$$
C_{n}^{\gamma}(x)=A_{n}^{\gamma}\left(1-x^{2}\right)^{-(2 \gamma+1) / 4} \sqrt{\dot{x}} e^{-\kappa \eta^{2} / 2}\left[H_{n}(\eta \sqrt{\kappa})+\bar{\varepsilon}\right]
$$

where $A_{n}^{\gamma}$ does not depend on $x$. Again, $|\bar{\varepsilon}|$ is small (in the sense described above) when $\kappa \rightarrow \infty$, uniformly with respect to $x \in(-1,1)$. By using

$$
C_{n}^{\gamma}(1)=\frac{(2 \gamma)_{n}}{n !}, \quad \lim _{z \rightarrow \infty} z^{-n} H_{n}(z)=2^{n}
$$


and the result in (2.18), we obtain

$$
A_{n}^{\gamma}=\sqrt{\nu}\left[2 c\left(x_{0}\right)\right]^{\gamma / 2-1 / 4} 2^{-n} \kappa^{-n / 2}(2 \gamma)_{n} / n ! .
$$

Evaluating this expression by using (2.19), we can write

$$
A_{n}^{\gamma}=\kappa^{-1 / 4} 2^{-n} e^{n / 2+1 / 4}(\gamma-1 / 2)^{\gamma}(\gamma+n / 2-1 / 4)^{-\gamma-n / 2+1 / 4}(2 \gamma)_{n} / n !,
$$

with $\kappa=n+\gamma$.

It is not difficult to verify that (2.21) contains (2.10) as a special case; from Olver's theory it follows that $\bar{\varepsilon}$ tends to zero in the limit considered.

\subsection{Other asymptotic forms for large $\gamma$ and/or $n$}

When in (2.5) $x$ runs in a domain that contains both the points $x_{0}$ and 1 , and $x_{0}$ may coalesce with the point 1 , the equation resembles a particular form of the Bessel equation. For this case we can use an LG transformation of the form

$$
\frac{\nu^{2}-\zeta}{4 \zeta^{2}}\left(\frac{d \zeta}{d x}\right)^{2}=\frac{x^{2}-x_{0}^{2}}{\left(1-x^{2}\right)^{2}}, \quad W(x)=\sqrt{-\dot{x}} w(\zeta),
$$

where $\nu$ is defined in (2.12), that is

$$
\nu=\sqrt{1-x_{0}^{2}}=\frac{\gamma-1 / 2}{\kappa}, \quad \kappa=n+\gamma,
$$

and the dot indicates differentiation with respect to $\zeta$. The point $x=x_{0}$ is transformed to $\zeta=\nu^{2}$, and the point $x=1$ to $\zeta=0$. The derivative $\dot{x}$ is negative in the domain of interest. Again, $\kappa$ is large, and $\gamma \geq 1 / 2$.

The relation between $x$ and $\zeta$ can be obtained by integrating the first of (2.23).

With these transformations the Gegenbauer equation (2.5) is transformed to

$$
\ddot{w}=\left[\kappa^{2}\left(\frac{\nu^{2}}{4 \zeta^{2}}-\frac{1}{4 \zeta}\right)-\frac{1}{4 \zeta^{2}}\right] w+\frac{\phi(\zeta)}{\zeta} w,
$$

where

$$
\frac{\phi(\zeta)}{\zeta}=-\dot{x}^{2} \frac{x^{2}+3}{4\left(1-x^{2}\right)^{2}}+\sqrt{-\dot{x}} \frac{d^{2}}{d \zeta^{2}} \frac{1}{\sqrt{-\dot{x}}}+\frac{1}{4 \zeta^{2}} .
$$

The function $\phi$ is analytic in a domain containing both the turning point $\zeta=\nu^{2}$ and the singular point $\zeta=0$.It can be verified that

$$
\phi(\zeta)=\mathcal{O}\left(\zeta^{-2}\right), \quad \text { as } \quad \zeta \rightarrow-\infty
$$


The solution of (2.24) can be put in the form $w(\zeta)=\sqrt{\zeta} J_{\gamma-1 / 2}(\kappa \sqrt{\zeta})+\varepsilon(\zeta)$. For the Gegenbauer polynomials we obtain

$$
C_{n}^{\gamma}(x)=B_{n}^{\gamma}\left(1-x^{2}\right)^{-(2 \gamma+1) / 4} \sqrt{-\dot{x}}\left[\sqrt{\zeta} J_{\gamma-1 / 2}(\kappa \sqrt{\zeta})+\varepsilon(\zeta)\right],
$$

where $B_{n}^{\gamma}$ does not depend on $x$. This number follows from the behavior of the $J$-Bessel function at $\zeta=0$ and $C_{n}^{\gamma}(1)=(2 \gamma)_{n} / n$ !. We also need the number $c_{1}$ in $x=1+c_{1} \zeta+$ $\mathcal{O}\left(\zeta^{2}\right)$ as $\zeta \rightarrow 0$, that is, $c_{1}=-1 /\left(1+x_{0}^{2}\right)$. So we obtain

$$
B_{n}^{\gamma}=\frac{(2 \gamma)_{n}}{n !} 2^{\gamma+1 / 2}\left(-c_{1}\right)^{\gamma / 2-1 / 4} \kappa^{1 / 2-\gamma} \Gamma(\gamma+1 / 2) .
$$

From Boyd \& DUNSTER (1986) it follows that (2.25) provides an asymptotic estimate of the Gegenbauer polynomial with $\kappa \rightarrow \infty, \gamma=\mathcal{O}(n)$. The estimate holds uniformly with respect to $x \in\left[-x_{0}+\delta, \infty\right)$ (and also in larger domains of the complex plane). Upper bounds for $|\varepsilon(\zeta)|$ follow from the cited reference, also for an estimate involving more terms in the expansion. Since the Gegenbauer polynomial is symmetric with respect to $x$, a similar expansion hold in the $x$-domain containing the points $-x_{0}$ and -1 .

A third expansion is needed to describe the behavior for $x \geq 1$ and $n=\mathcal{O}(\gamma)$. As explained in Olver( (1974), for this case an expansion can be given in terms of elementary functions.

\section{LAGUerRe POLYNOMIALS}

The Laguerre polynomials have the explicit representation

$$
L_{n}^{\alpha}(x)=\sum_{k=0}^{n}\left(\begin{array}{l}
n+\alpha \\
n-k
\end{array}\right) \frac{(-x)^{k}}{k !} .
$$

We assume that the parameters are real with $n \geq 0, \alpha \geq 0, x \in \mathbb{R}$. For large values of

$$
\kappa=n+1 / 2(\alpha+1)
$$

the asymptotic behavior can be described in terms of the Hermite polynomial and the $J$-Bessel function, just as in the previous section. In this section we summarize the recent results obtained in TEMME (1986). The results also follow from earlier results in the literature for Whittaker functions. The relation between Laguerre polynomials and Whittaker functions is

$$
L_{n}^{\alpha}(z)=\frac{(-1)^{n}}{n !} z^{-(\alpha+1) / 2} e^{z / 2} W_{\kappa, \mu}(z)=\frac{\Gamma(\alpha+n+1)}{n ! \Gamma(\alpha+1)} z^{-(\alpha+1) / 2} e^{z / 2} M_{\kappa, \mu}(z),
$$


with $\kappa$ as in (3.1) and $\mu=\alpha / 2$. The functions $M_{\kappa, \mu}(z), W_{\kappa, \mu}(z)$ are solutions of Whittaker's equation

$$
\frac{d^{2} y}{d z^{2}}=\left[\frac{1}{4}-\frac{\kappa}{z}+\frac{\mu^{2}-1 / 4}{z^{2}}\right] y
$$

A first transformation $z=4 \kappa x$ yields the differential equation

$$
W^{\prime \prime}=\kappa^{2}\left[\frac{4\left(x-x_{1}\right)\left(x-x_{2}\right)}{x^{2}}-\frac{1}{4 x^{2}}\right] W,
$$

where

$$
x_{1}=\frac{1-\sqrt{1-\tau^{2}}}{2}, \quad x_{2}=\frac{1+\sqrt{1-\tau^{2}}}{2}, \quad \tau=\frac{\alpha}{2 \kappa}
$$

with solution

$$
W(x)=x^{(\alpha+1) / 2} e^{-2 \kappa x} L_{n}^{\alpha}(4 \kappa x) .
$$

The $x$-zeros of the polynomial $L_{n}^{\alpha}(4 \kappa x)$ occur within the interval $\left[x_{1}, x_{2}\right]$. When $\alpha>>n$ the parameter $\tau$ tends to unity, and the turning points $x_{1}, x_{2}$ are close together. This case is described by a parabolic cylinder function, which reduces for this particular case of the Laguerre polynomials to a Hermite polynomial. When $\tau \rightarrow 0$ the zeros of $L_{n}^{\alpha}(4 \kappa x)$ are spread over the $x$-interval $(0,1)$, and a $J$-Bessel function is used in this case.

\subsection{Estimation in terms of a Hermite polynomial}

The LG transform is defined by

$$
\left(\eta^{2}-\rho^{2}\right)\left(\frac{d \eta}{d x}\right)^{2}=\frac{4\left(x-x_{1}\right)\left(x-x_{2}\right)}{x^{2}}, \quad W(x)=\sqrt{\dot{x}} w(\eta),
$$

where the dot indicates differentiation with respect to $\eta$, and $\rho$ is given by

$$
\rho=\sqrt{2(1-\tau)} .
$$

The relation between $\eta$ and $x$ is one-to-one, with

$$
\eta(0)=-\infty, \quad \eta\left(x_{1}\right)=-\rho, \quad \eta\left(x_{2}\right)=\rho, \quad \eta(+\infty)=\infty .
$$

The differential equation (3.4) transforms into

$$
\ddot{w}=\kappa^{2}\left(\eta^{2}-\rho^{2}\right) w+\psi(\eta) w,
$$

where

$$
\psi(\eta)=\frac{3 \eta^{2}+2 \rho^{2}}{4\left(\eta^{2}-\rho^{2}\right)^{2}}-\frac{x\left(\eta^{2}-\rho^{2}\right)}{64\left[\left(x-x_{1}\right)\left(x-x_{2}\right)\right]^{3}}\left(4 x^{3}+\left(1-4 \tau^{2}\right) x+\tau^{2}\right) .
$$


We have

$$
\psi(\eta)=\mathcal{O}\left(\frac{1}{\eta^{2}+1}\right), \quad \eta \in \mathbb{R},
$$

uniformly with respect to $\tau \in\left[\tau_{0}, 1\right]$, where $\tau_{0}$ is a fixed number in $(0,1)$.

By identifying solutions, and considering the asymptotic behavior of the Laguerre polynomials and the Hermite polynomials, we arrive at the estimate

$$
\begin{aligned}
L_{n}^{\alpha}(4 \kappa x)= & \frac{(-1)^{n}}{n !} 2^{-\alpha-n / 2-3 / 4} \kappa^{-\alpha / 2-1 / 4} x^{-\alpha / 2}\left(\frac{n+\alpha+1 / 2}{e}\right)^{n+\alpha+1 / 2} \\
& e^{2 \kappa x-\kappa \eta^{2} / 2} \sqrt{\dot{x}}\left[H_{n}(\eta \sqrt{\kappa})+\bar{\varepsilon}\right]
\end{aligned} .
$$

This representation has an asymptotic interpretation as $\kappa \rightarrow \infty$; in that case $|\bar{\varepsilon}|$ is small (in the same sense as in (2.21)), uniformly with respect to $x \in(0, \infty)$ and $t \in\left[\tau_{0}, 1\right]$. The latter gives for $\alpha$ the condition

$$
\alpha \geq \frac{\tau_{0}}{1-\tau_{0}}(2 n+1)
$$

with $\tau_{0}$ any fixed number in $(0,1)$.

\subsection{Other asymptotic forms for large $\alpha$ and/or $n$.}

In TEMME (1986) details are given for two other asymptotic forms of the Laguerre polynomials, one in terms of the $J$-Bessel function, and one in terms of an Airy function. The Bessel function is used to describe the asymptotics of $L_{n}^{\alpha}(4 \kappa x)$ for $\kappa \rightarrow \infty$, uniformly with respect to $\alpha \in\left[0, n_{1} n\right]$ and $x \in\left(-\infty, x_{0}\right], x_{0}=\xi_{0} x_{1}+\left(1-\xi_{0}\right) x_{2}$, where $x_{1,2}$ are given in (3.5), $n_{1}$ is a fixed positive number, and $\xi_{0}$ is a fixed number in $(0,1)$. The Airy function is used to describe the asymptotics uniformly with respect to $\alpha \in\left[0, n_{2} n\right]$ and $x \in\left[x_{3}, \infty\right), x_{3}=\xi_{3} x_{1}+\left(1-\xi_{3}\right) x_{2}$, with $n_{2}$ a fixed positive number, and $\xi_{3}$ a fixed number in $(0,1)$.

Both forms follow from Dunster (1989), where the results are obtained in the setting of Whittaker functions. A fourth form can be given in terms of elementary functions; it is uniformly valid with respect to $x \leq 0$ and $\alpha \in\left[n_{3} n, \infty\right)$, where $n_{3}$ is a fixed positive number.

In this way we have four asymptotic estimates in overlapping domains of the three real parameter domains $x \in \mathbb{R}, n \geq 0, \alpha \geq 0$. It is not difficult to verify that $\alpha$ may assume negative values, as long as $\kappa \rightarrow \infty$. 


\section{JACOBI POLYNOMIALS}

The Jacobi polynomials $P_{n}^{(\alpha, \beta)}(x)$ are solutions of the differential equation

$$
\left(1-x^{2}\right) y^{\prime \prime}+[(\beta-\alpha)-(\alpha+\beta+2) x] y^{\prime}+n(n+\alpha+\beta+1) y=0
$$

In fact this is a differential equation of hypergeometric type, the relation with hypergeometric functions being

$$
P_{n}^{(\alpha, \beta)}(x)=\left(\begin{array}{c}
n+\alpha \\
n
\end{array}\right) F\left(-n, \alpha+\beta+n+1 ; \alpha+1 ; \frac{1-x}{2}\right)
$$

which gives the explicit representation

$$
P_{n}^{(\alpha, \beta)}(x)=\frac{\Gamma(\alpha+n+1)}{n ! \Gamma(\alpha+\beta+n+1)} \sum_{k=0}^{n}\left(\begin{array}{l}
n \\
k
\end{array}\right) \frac{\Gamma(\alpha+\beta+n+k+1)}{\Gamma(\alpha+k+1)}\left(\frac{x-1}{2}\right)^{k} .
$$

The following normalization and symmetry relations hold

$$
P_{n}^{(\alpha, \beta)}(1)=\left(\begin{array}{c}
n+\alpha \\
n
\end{array}\right), \quad P_{n}^{(\alpha, \beta)}(-x)=(-1)^{n} P_{n}^{(\beta, \alpha)}(x) .
$$

The Gegenbauer polynomial is a special case of the Jacobi polynomial:

$$
C_{n}^{\gamma}(x)=\frac{(2 \gamma)_{n}}{(\gamma+1 / 2)_{n}} P_{n}^{(\gamma-1 / 2, \gamma-1 / 2)}(x)
$$

The function

$$
W(x)=(1-x)^{(\alpha+1) / 2}(1+x)^{(\beta+1) / 2} P_{n}^{(\alpha, \beta)}(x)
$$

satisfies the equation

$$
W^{\prime \prime}=\frac{Q(x, \alpha, \beta, n)}{\left(1-x^{2}\right)^{2}} W
$$

with

$$
\begin{gathered}
Q(x, \alpha, \beta, n)=\kappa^{2}\left(x^{2}-1\right)+1 / 2 x\left(\alpha^{2}-\beta^{2}\right)+1 / 2\left(\alpha^{2}+\beta^{2}\right)-1 / 4\left(3+x^{2}\right), \\
\kappa=n+(\alpha+\beta+1) / 2 .
\end{gathered}
$$

We factorize the function $Q$ in the following way

$$
Q(x, \alpha, \beta, n)=\kappa^{2}\left(x-x_{1}\right)\left(x-x_{2}\right)-1 / 4\left(3+x^{2}\right)
$$

where

$$
x_{1,2}=\frac{\beta^{2}-\alpha^{2} \pm \sqrt{\left[4 \kappa^{2}-(\alpha+\beta)^{2}\right]\left[4 \kappa^{2}-(\alpha-\beta)^{2}\right]}}{4 \kappa^{2}}
$$


where $x_{2}$ has the + sign. It is not difficult to verify that this factorization yields the same equations for the Gegenbauer polynomials in the case that $\alpha=\beta=\gamma-1 / 2$.

We consider the case where the parameters $\alpha, \beta$ are positive, and the independent variable $x$ is real. The parameter $\kappa$ of (4.8) is large.

The numbers $x_{1,2}$ satisfy $-1<x_{1}<x_{2}<1$. Several limiting cases of these numbers are possible. To describe some of the phenomena, we introduce

$$
\sigma=\frac{\alpha+\beta}{2 \kappa}, \quad \delta=\frac{\alpha-\beta}{2 \kappa} .
$$

Then we can write

$$
x_{1,2}=-\sigma \delta \pm \sqrt{\left(1-\sigma^{2}\right)\left(1-\delta^{2}\right)}
$$

and we have the following cases:

- when $n>>\alpha+\beta$, that is, $\sigma \rightarrow 0$, then $x_{1} \rightarrow-1, x_{2} \rightarrow+1$;

- when $\alpha+\beta>>n$, that is, $\sigma \rightarrow 1$, then $x_{1}$ and $x_{2}$ coalesce at $-\delta$;

- when $\alpha>>\beta$, that is, $\delta \rightarrow 1$, then $x_{1}$ and $x_{2}$ coalesce at $-\sigma$;

- when $\beta>>$, that is, $\delta \rightarrow-1$, then $x_{1}$ and $x_{2}$ coalesce at $\sigma$.

Note that the collisions may happen at +1 when $\beta>>>>n$, and at -1 when $\alpha>>\beta>>n$.

In the following subsections we give the setup of two LG transformations for obtaining asymptotic estimates of the Jacobi polynomials in terms of the Gegenbauer polynomials and of the Laguerre polynomials. Both forms seem to be new, but the proofs of the asymptotic nature of the approximations will be elaborated elsewhere. We demonstrate the usefulness of the estimates by comparing the zeros of the Jacobi polynomials with transformed zeros of the LG approximants for these cases: the Gegenbauer polynomials and the Laguerre polynomials.

\subsection{Estimation in terms of a Gegenbauer polynomial}

We define a Liouville-Green transformation for (4.7) by writing

$$
\frac{\eta^{2}-\rho^{2}}{\left(1-\eta^{2}\right)^{2}}\left(\frac{d \eta}{d x}\right)^{2}=\frac{\left(x-x_{2}\right)\left(x-x_{1}\right)}{\left(1-x^{2}\right)^{2}}, \quad W(x)=\sqrt{\dot{x}} w(\eta),
$$

where the dot indicates differentiation with respect to $\eta$, and $\rho$ is the non-negative number defined by

$$
\int_{-\rho}^{\rho} \frac{\sqrt{\rho^{2}-\eta^{2}}}{1-\eta^{2}} d \eta=\int_{x_{1}}^{x_{2}} \frac{\sqrt{\left(x_{2}-x\right)\left(x-x_{1}\right)}}{1-x^{2}} d x .
$$


Evaluation of both integrals yields

$$
\pi\left(1-\sqrt{1-\rho^{2}}\right)=\pi\left[1-1 / 2 \sqrt{\left(1-x_{1}\right)\left(1-x_{2}\right)}-1 / 2 \sqrt{\left(1+x_{1}\right)\left(1+x_{2}\right)}\right] .
$$

The square roots in the right-hand side can easily be evaluated; using (4.10) we obtain

$$
\sqrt{\left(1-x_{1}\right)\left(1-x_{2}\right)}=\frac{\alpha}{\kappa}, \quad \sqrt{\left(1+x_{1}\right)\left(1+x_{2}\right)}=\frac{\beta}{\kappa} .
$$

It follows that

$$
\sqrt{1-\rho^{2}}=\frac{\alpha+\beta}{2 \kappa}
$$

The relation between $\eta$ and $x$ is one-to-one, with the points $x_{1}$ and $x_{2}$ corresponding respectively to $-\rho$ and $\rho$. For the integration of the first of (4.11) we introduce the following notations

$$
\begin{gathered}
x_{0}=1 / 2\left(x_{2}-x_{1}\right), \quad \bar{x}=1 / 2\left(x_{1}+x_{2}\right), \quad \rho=\sin \mu, \quad \nu=\cos \mu, \\
T= \begin{cases}\arccos \left[(x-\bar{x}) / x_{0}\right], & \text { if } x_{1}<x<x_{2} ; \\
\operatorname{arccosh}\left[(x-\bar{x}) / x_{0}\right], & \text { if } x_{2}<x<1 ; \\
\operatorname{arccosh}\left[(\bar{x}-x) / x_{0}\right], & \text { if }-1<x<x_{1} .\end{cases}
\end{gathered}
$$

We also use

$$
R= \begin{cases}\sqrt{\left(x_{2}-x\right) /\left(x-x_{1}\right)}, & \text { if } x_{1}<x<x_{2} ; \\ \sqrt{\left(x-x_{2}\right) /\left(x-x_{1}\right)}, & \text { if } x_{2}<x<1 ; \\ \sqrt{\left(x_{1}-x\right) /\left(x_{2}-x\right)}, & \text { if }-1<x<x_{1} .\end{cases}
$$

Integrating the first of (4.11) we obtain when $x_{1} \neq x_{2}$, that is, $x_{0} \neq 0$

(i) $-\rho \leq \eta \leq \rho, \quad x_{1} \leq x \leq x_{2}$ :

$$
\begin{aligned}
& \arcsin \frac{\eta}{\rho}-\nu \arctan \frac{\nu \eta}{\sqrt{\rho^{2}-\eta^{2}}}-1 / 2 \pi(1+\nu)= \\
& -T-\frac{\beta}{\kappa} \arctan \frac{\kappa\left(1+x_{2}\right)}{\beta R}-\frac{\alpha}{\kappa} \arctan \frac{\kappa\left(1-x_{2}\right)}{\alpha R}
\end{aligned}
$$

(ii) $\rho \leq \eta<1, \quad x_{2} \leq x<1$ :

$$
\begin{aligned}
& -\operatorname{arccosh} \frac{\eta}{\rho}+\nu \operatorname{arctanh} \frac{\sqrt{\eta^{2}-\rho^{2}}}{\nu \eta}= \\
& -T+\frac{1}{2 \kappa}\left[\beta \ln \frac{\kappa\left(1+x_{2}\right)+\beta R}{\kappa\left(1+x_{2}\right)-\beta R}+\alpha \ln \frac{\kappa\left(1-x_{2}\right)+\alpha R}{\kappa\left(1-x_{2}\right)-\alpha R}\right]
\end{aligned}
$$


(iii) $-1<\eta \leq-\rho, \quad-1<x \leq x_{1}$ :

$$
\begin{aligned}
& -\operatorname{arccosh} \frac{\eta}{\rho}+\nu \operatorname{arctanh} \frac{\nu \eta}{\sqrt{\eta^{2}-\rho^{2}}}= \\
& -T-\frac{1}{2 \kappa}\left[\beta \ln \frac{\kappa\left(1+x_{1}\right)+\beta R}{\kappa\left(1+x_{1}\right)-\beta R}-\alpha \ln \frac{\kappa\left(1-x_{1}\right)+\alpha R}{\kappa\left(1-x_{1}\right)-\alpha R}\right] .
\end{aligned}
$$

Alternatively, when $x_{1}=x_{2}=\bar{x}$, that is, $x_{0}=0$, so that $\rho=0$, we find for $|\eta|<1,|x|<1$

$$
\ln \left(1-\eta^{2}\right)=(1-\bar{x}) \ln \frac{1-x}{1-\bar{x}}+(1+\bar{x}) \ln \frac{1+x}{1+\bar{x}}
$$

with $\operatorname{sign}(\eta)=\operatorname{sign}(x-\bar{x})$. Whether or not $x_{0}=0$, the differential equation (4.7) transforms into

$$
\ddot{w}=\left[\kappa^{2} \frac{\left(\eta^{2}-\rho^{2}\right)}{\left.1-\eta^{2}\right)^{2}}-\frac{\eta^{2}+3}{4\left(1-\eta^{2}\right)^{2}}\right] w+\psi(\eta) w,
$$

where

$$
\psi(\eta)=-\dot{x}^{2} \frac{x^{2}+3}{4\left(1-x^{2}\right)^{2}}+\sqrt{\dot{x}} \frac{d^{2}}{d \eta^{2}} \frac{1}{\sqrt{\dot{x}}}+\frac{\eta^{2}+3}{4\left(1-\eta^{2}\right)^{2}} .
$$

For identifying the solutions we need to know the asymptotic behavior of $x(\eta)$ as $\eta \rightarrow 1$. It is straightforward to verify from (4.16) that

$$
1-x=(1-\eta)^{(\alpha+\beta) /(2 \alpha)} C[1+o(1)], \quad \text { as } \quad \eta \uparrow 1,
$$

where $C$ is not depending on $\eta$, and is given by ( $t$ is given in (4.14) with $x=1$ )

$$
\begin{gathered}
C=\exp \left[-\frac{2 \kappa T}{\alpha}+\frac{\beta}{\alpha} \ln \frac{\sqrt{\left(1+x_{2}\right)\left(1-x_{1}\right)}+\sqrt{\left(1+x_{1}\right)\left(1-x_{2}\right)}}{\sqrt{\left(1+x_{2}\right)\left(1-x_{1}\right)}-\sqrt{\left(1+x_{1}\right)\left(1-x_{2}\right)}}+\right. \\
\left.\ln \left\{\frac{2\left(1-x_{1}\right)\left(1-x_{2}\right)}{x_{2}-x_{1}}\left(\sqrt{1-x_{1}}+\sqrt{1-x_{2}}\right)\right\}\right] .
\end{gathered}
$$

When $\psi=0$ the solution of (4.18) is a Gegenbauer polynomial (see (2.5)); that is,

$$
w_{0}(\eta)=\left(1-\eta^{2}\right)^{(2 \gamma+1) / 4} C_{n}^{\gamma}(\eta)
$$

where $\gamma=(\alpha+\beta+1) / 2$. We write for the complete solution of (4.18)

$$
w(\eta)=\left(1-\eta^{2}\right)^{(2 \gamma+1) / 4} C_{n}^{\gamma}(\eta)+\varepsilon(\eta),
$$

with the expectation that $\varepsilon(\eta)$ is a small contribution as $\kappa \rightarrow \infty$. Using (2.4), (2.5) we obtain the asymptotic estimate

$$
P_{n}^{(\alpha, \beta)}(x)=A_{n}^{\alpha, \beta} \sqrt{\dot{x}}(1-x)^{-(\alpha+1) / 2}(1+x)^{-(1+\beta) / 2}\left(1-\eta^{2}\right)^{(2 \gamma+1) / 4}\left[C_{n}^{\gamma}(\eta)+\bar{\varepsilon}(\eta)\right]
$$


where $A_{n}^{\alpha, \beta}$ can be derived by computing the behavior of the polynomials at $x=1, \eta=1$ from (2.22) and (4.4). The result is

$$
A_{n}^{\alpha, \beta}=2^{(\beta-\alpha) / 4} C^{\alpha / 2} \sqrt{\frac{2 \alpha}{\alpha+\beta}} \frac{\Gamma(2 \gamma) \Gamma(n+\alpha+1)}{\Gamma(\alpha+1) \Gamma(n+2 \gamma)} .
$$

When $\alpha=\beta$, the functions $\psi, \varepsilon, \bar{\varepsilon}$ vanish identically; the mappings described in (4.15), (4.16), and (4.17) reduce to the identity mapping $x=\eta$, and the relation between the polynomials is then as in (4.5).

\subsection{Estimation in terms of a Laguerre polynomial}

A known limit is

$$
\lim _{\alpha \rightarrow \infty} P_{n}^{(\alpha, \beta)}(2 x / \alpha-1)=(-1)^{n} L_{n}^{\beta}(x) .
$$

We give a new asymptotic estimate that includes this relation. We concentrate on an estimate that can describe the behavior of $P_{n}^{(\alpha, \beta)}(x)$ for $x$-values in a domain that contains the singular point $x=-1$ (a double pole) of (4.7) and both turning points $x_{1,2}$ defined in $(4.10)$ and located in $(-1,1)$. Recall that both turning points coalesce with the pole when $\alpha>>\beta>n$. Especially this situation can be described by the Laguerre polynomial.

We define an LG transformation by writing $W(x)=\sqrt{\dot{x}} w(\eta)$ and

$$
\frac{4\left(\eta-\rho_{1}\right)\left(\eta-\rho_{2}\right)}{\eta^{2}}\left(\frac{d \eta}{d x}\right)^{2}=\frac{\left(x-x_{2}\right)\left(x-x_{1}\right)}{\left(1-x^{2}\right)^{2}},
$$

where the positive numbers $\rho_{1,2}$ are to be determined. A first condition reads

$$
\begin{aligned}
\int_{\rho_{1}}^{\rho_{2}} & \frac{\sqrt{\left(\rho_{2}-\eta\right)\left(\eta-\rho_{1}\right)}}{\eta} d \eta= \\
& \int_{x_{1}}^{x_{2}} \frac{\sqrt{\left(x_{2}-x\right)\left(x-x_{1}\right)}}{1-x^{2}} d x .
\end{aligned}
$$

Evaluation of both integrals gives

$$
\rho_{1}+\rho_{2}-2 \sqrt{\rho_{1} \rho_{2}}=\frac{2 n+1}{2 \kappa} .
$$

It is required that the relation between $\eta$ and $x$ is one-to-one for $x \in(-\infty, 1)$, with the points $x_{1}$ and $x_{2}$ corresponding respectively to $\rho_{1}$ and $\rho_{2}$. We also request that the mapping is regular at the corresponding points $x=-1, \eta=0$. A necessary condition follows from $\eta /(1+x)=\mathcal{O}(1)$ as $x \rightarrow-1$. Assuming that

$$
\frac{d \eta}{d x} \sim \frac{\eta}{1+x} \rightarrow L, \quad \text { as } \quad x \rightarrow-1,
$$


we obtain from (4.22) the second condition

$$
4 \sqrt{\rho_{1} \rho_{2}}=\frac{\beta}{\kappa},
$$

where we used the second of (4.12). From those two conditions $\rho_{1,2}$ can be determined. We have

$$
2 \sqrt{\rho_{1,2}}=\sqrt{(2 n+1+2 \beta) / 2 \kappa} \pm \sqrt{(2 n+1) / 2 \kappa},
$$

where $\rho_{2}$ has the + sign.

The integrated form of (4.22) for the location of the parameters $\rho_{1} \leq \eta \leq \rho_{2}, x_{1} \leq$ $x \leq x_{2}$ reads

$$
\begin{aligned}
& 2 S-2 \sqrt{\rho_{1} \rho_{2}} \arcsin \frac{\left(\rho_{1}+\rho_{2}\right) \eta-2 \rho_{1} \rho_{2}}{\eta\left(\rho_{2}-\rho_{1}\right)}-\left(\rho_{1}+\rho_{2}\right) \arcsin \frac{\rho_{1}+\rho_{2}-2 \eta}{\rho_{2}-\rho_{1}}= \\
& -T-\frac{\beta}{\kappa} \arctan \frac{\kappa\left(1+x_{2}\right)}{\beta R}-\frac{\alpha}{\kappa} \arctan \frac{\kappa\left(1-x_{2}\right)}{\alpha R}-\left(\frac{2 n+1}{4 \kappa}-1\right) \pi,
\end{aligned}
$$

where $S=\sqrt{\left(\rho_{2}-\eta\right)\left(\eta-\rho_{1}\right)}$ and the parameters of the right-hand side are as in (4.15).

The LG transformation (4.22) transforms (4.7) into the differential equation

$$
\ddot{w}=\left[\kappa^{2} \frac{4\left(\eta-\rho_{1}\right)\left(\eta-\rho_{2}\right)}{\eta^{2}}-\frac{1}{4 \eta^{2}}\right] w+\psi(\eta) w
$$

where

$$
\psi(\eta)=-\dot{x}^{2} \frac{x^{2}+3}{4\left(1-x^{2}\right)^{2}}+\sqrt{\dot{x}} \frac{d^{2}}{d \eta^{2}} \frac{1}{\sqrt{\dot{x}}}+\frac{1}{4 \eta^{2}} .
$$

When $\psi=0$ we denote the solution by $w_{0}$. This function can be written in terms of the Whittaker functions, just as the solution of the differential equation in (3.4), but now with parameters $\rho_{1,2}$ satisfying (4.23) and (4.24). To obtain the proper form of the solutions, we transform the above differential equation (with $\psi=0$ ) into (3.3), with $4 \kappa \eta$ replaced with $z$. Then we obtain

$$
\frac{d^{2} y}{d z^{2}}=\left[\frac{1}{4}-\frac{k}{z}+\frac{m^{2}-1 / 4}{z^{2}}\right] y
$$

with solutions the Whittaker functions $W_{k, m}(z), M_{k, m}(z)$, with

$$
k=\kappa\left(\rho_{1}+\rho_{2}\right)=n+\frac{\beta+1}{2}, \quad m=2 \kappa \sqrt{\rho_{1} \rho_{2}}=1 / 2 \beta .
$$

It follows that when $\psi=0$ a solution of $(4.26)$ is

$$
w_{0}(\eta)=\eta^{\beta / 2} e^{-2 \kappa \eta} L_{n}^{\beta}(4 \kappa \eta)
$$


We write for the complete solution

$$
w(\eta)=\eta^{\beta / 2} e^{-2 \kappa \eta} L_{n}^{\beta}(4 \kappa \eta)+\varepsilon(\eta)
$$

with the expectation that $\varepsilon(\eta)$ is a small contribution as $\kappa \rightarrow \infty$. We obtain an asymptotic estimate of the Jacobi polynomial in terms of the Laguerre polynomial

$$
P_{n}^{(\alpha, \beta)}(x)=B_{n}^{\alpha, \beta} \sqrt{\dot{x}}(1-x)^{-(\alpha+1) / 2}(1+x)^{-(1+\beta) / 2} \eta^{\beta / 2} e^{-2 \kappa \eta}\left[L_{n}^{\beta}(4 \kappa \eta)+\bar{\varepsilon}(\eta)\right]
$$

where $B_{n}^{\alpha, \beta}$ can be derived by computing the behavior of the polynomials at $x=$ $-\infty, \eta=-\infty$.

We expect that the above approximation holds for $\kappa \rightarrow \infty$, uniformly with respect to $x \in[-\infty, 1-\delta]$, and $x_{1}, x_{2} \in[-1,1-\delta]$, with $\delta$ a small fixed positive number.

\section{NUMERICAL VERIFICATION BY COMPUTATION OF ZEROS}

For given values of $n, \alpha, \beta$ we have verified the asymptotic estimate (4.28), where the relation between $\eta$ and $x$ is given in (4.25). We computed the zeros $z_{m}, m=1 \ldots n$ of the Laguerre polynomial $L_{n}^{\beta}(z)$, giving $\eta_{m}=z_{m} /(4 \kappa), m=1 \ldots n$. By inverting (4.25) we obtained $x_{m}=x\left(\eta_{m}\right)$, and we compared these values with "exact" zeros $x_{m}^{(e)}$ of the Jacobi polynomial $P_{n}^{(\alpha, \beta)}(x)$. In Table 5.1 we show for $n=10, \beta=5.5$ and several values of $\alpha$ the corresponding number of correct decimal digits in the approximation of the zeros. That is, we show

$$
\log _{10}\left|x_{m}^{(e)}-x_{m}\right|, \quad m=1,2, \ldots 10
$$

\begin{tabular}{|c|c|c|c|c|c|c|c|c|c|}
\hline & $\alpha$ & 1.0 & 2.5 & 5.0 & 10 & 25 & 50 & 75 & 100 \\
\hline \multicolumn{10}{|l|}{$m$} \\
\hline 1 & & 4.3 & 4.5 & 4.7 & 5.0 & 5.6 & 6.3 & 6.7 & 1.0 \\
\hline 2 & & 4.1 & 4.2 & 4.4 & 4.7 & 5.3 & 6.0 & 6.4 & \\
\hline 3 & & 3.9 & 4.0 & 4.2 & 4.5 & 5.2 & 5.8 & 6.2 & \\
\hline 4 & & 3.8 & 3.9 & 4.1 & 4.4 & 5.0 & 5.6 & 6.1 & \\
\hline 5 & & 3.7 & 3.8 & 4.0 & 4.3 & 4.9 & 5.5 & 5.9 & \\
\hline 6 & & 3.6 & 3.7 & 3.9 & 4.2 & 4.8 & 5.4 & 5.8 & \\
\hline 7 & & 3.6 & 3.7 & 3.9 & 4.2 & 4.7 & 5.3 & 5.7 & \\
\hline 8 & & 3.6 & 3.7 & 3.9 & 4.1 & 4.7 & 5.3 & 5.6 & \\
\hline 9 & & 3.6 & 3.7 & 3.9 & 4.1 & 4.6 & 5.2 & 5.6 & \\
\hline 10 & & 3.7 & 3.8 & 4.0 & 4.2 & 4.6 & 5.1 & 5.5 & \\
\hline
\end{tabular}

Table 5.1. Correct decimal digits in the approximations of zeros of $P_{10}^{(\alpha, 5.5)}(x)$ 
The values of $z_{m}$ and $x_{m}^{(e)}$ are obtained from a numerical procedure that generates the zeros by computing the eigenvalues of a tri-diagonal matrix that corresponds with the recursion formulas of the polynomials.

From the table it follows that the zeros near $x=-1$ are slightly better approximated, but that in general the approximations are uniform with respect to $m$. A similar table for the asymptotic estimate (3.8) is given in [18], showing the same sharp results. We also used the numerical verification for the asymptotic estimates (2.21) and (4.21), again with satisfactory results.

Acknowledgment The author wants to thank Drs. Adri Olde Daalhuis (CWI) for his comments on earlier versions of the paper.

\section{REFERENCES}

[1] R. Askey (1982), The collected works of Gabor Szegö, Vol. 2, Birkhauser.

[2] W.G.C. Boyd \& T.M. Dunster (1986), Uniform asymptotic solution of a class of second order linear differential equations having a turning point and a regular singularity, with an application to Legendre functions, SIAM J. Math. Anal. 17, 422-450.

[3] T.M. Dunster (1989), Uniform asymptotic expansions for Whittaker's confluent hypergeometric functions, SIAM J. Math. Anal. 20, 744-760.

[4] D. Elliot (1971), Uniform asymptotic expansions of the Jacobi polynomials and an associated function, Math. Comp. 25, 309-315.

[5] A. Erdélyi, (1960), Asymptotic forms for Laguerre polynomials, J. Indian Math. Soc. 24, 235-250.

[6] C.L. Frenzen \& R. Wong (1985), A uniform asymptotic expansions of the Jacobi polynomials with error bounds, Can. J. Math. 37, 979-1007.

[7] C.L. Frenzen \& R. Wong (1988), Uniform asymptotic expansions of Laguerre polynomials, SIAM J. Math. Anal. 19, 1232-1248.

[8] Li-Chen Chen \& M.E.H. Ismail (1989), On asymptotics of Jacobi polynomials, Report ICM 89-004, Department of Mathematics, University of south Florida, Tampa.

[9] H.N. Mhaskar \& E.B. Saff (1984), Polynomials with Laguerre weights in $L^{p}$, 511-523 in Rational approximation and interpolation, P.R. Graves-Morris \& E.B. Saff (eds.), LNM 1105, Springer-Verlag.

[10] D.S. Moak, E.B. SAFF \& R.S. VARGA (1979), On the zeros of Jacobi polynomials $P_{n}^{\left(\alpha_{n}, \beta_{n}\right)}(x)$, Trans. Amer. Math. Soc. 249 159-163. 
[11] J.J. NESTOR III (1984), Uniform asymptotic approximations of solutions of secondorder linear differential equations, with a coalescing simple turning point and simple pole. Ph.D. thesis, Applied Mathematics, University of Maryland.

[12] F.W.J. Olver (1974), Asymptotics and Special Functions, Academic Press, New York.

[13] F.W.J. Olver (1975-a), Second-order linear differential equations with two turning points, Phil. Trans. Roy. Soc. London, Ser. A 278, 175-185.

[14] F.W.J. OLVER (1975-b), Unsolved problems in the asymptotic estimation of special functions, 99-142 in Theory and application of special functions, R. Askey (ed.), Academic Press.

[15] F.W.J. Olver (1980), Whittaker functions with both parameters large: uniform approximations in terms of parabolic cylinder functions, Proc. Royal Soc. Edinb. 84A, 213-234.

[16] E.B. SAFF \& R.S. VARGA (1979), The sharpness of Lorentz's theorem on incomplete polynomials, Trans. Amer. Math. Soc. 249 163-186.

[17] G. SzEgö (1958), Orthogonal polynomials, Amer. Math. Soc. Colloq. Publ., Vol. 23, New York.

[18] N.M. Temme (1986), Laguerre polynomials: asymptotics for large degree, CWI Report AM-R8610 (submitted). 